\title{
Estudo da fotodegradação de poliisoprenos naturais (borrachas naturais) em radiações específicas do ultravioleta (UV)
}

\author{
Kárin Astrid Marques dos Santos ${ }^{1}$
}

\begin{abstract}
RESUMO - Filmes de poliisoprenos (PI) naturais e sintéticos foram expostos à luz ultravioleta (UV) e analisados por espectroscopia de absorção UV, antes e após exposição à radiação UV. Filmes de padrões cis PII foram irradiados separadamente por fontes de luz com comprimentos de ondas iguais a 253 e 300 . A fotooxidação foi monitorada observando-se a dependência das intensidades das absorções correspondentes aos modos de estiramento $\mathrm{C}=\mathrm{C}, \mathrm{C}=\mathrm{O}$ e $\mathrm{O}-\mathrm{H}$ e do modo "wagging" =C-H de cis-e trans-PI em função do tempo de exposição. A irradiação em $253 \mathrm{~nm}$ levou a reações de reticulação do polímero, impedindo a completa oxidação do filme polimérico. Para irradiação em 300nm, as reações de cisão da cadeia ocorreram preferencialmente, levando à completa oxidação do filme polimérico. Os elastômeros de Hevea brasilienses (HB) e Hancornia speciosa (HS) foram caracterizadas por espectroscopia de absorção no infravermelho (FTIR) como cis-1,4-poliisopreno. A foto-oxidação dos elastômeros apresentou cinética diferente com tempo de indução menor que o observado para os padrões cis-PI. A maior velocidade para o processo de fotodegradação dos filmes de BNs foi atribuída à presença de impurezas, caracterizadas por bandas de absorção nas regiões de estiramento $\mathrm{O}-\mathrm{H}$ e $\mathrm{C}=\mathrm{O}$, mesmo antes da exposição à radiação $\mathrm{UV}$.
\end{abstract}

Palavras-chave: foto-oxidação; poliisoprenos; FTIR; borracha natural.

\section{Study photo-degradation of natural polyisoprenes (natural rubber) at specific UV radiations}

\begin{abstract}
In this work, synthetic and natural polyisoprenes (PI) films were exposed to different UV light sources and then analyzed by FTIR and UV spectroscopy, before and after exposure to the UV source. Films of the PI standards were separately irradiated by UV sources of different wavelengths, 253,300 , and $350 \mathrm{~nm}$. The photo-oxidation was monitored by looking at the dependence of the IR absorption intensities of the $\mathrm{C}=\mathrm{C}, \mathrm{C}=\mathrm{O}$, and $\mathrm{O}-\mathrm{H}$ stretching and the $=\mathrm{C}-\mathrm{H}$ wagging modes of cis- and trans-PI on the exposure time. Irradiation at $253 \mathrm{~nm}$ leads to cross-linking and polymer reticulation avoiding the complete oxidation of the polymer film. Under UV irradiation at $300 \mathrm{~nm}$ the chain scission reactions
\end{abstract}

${ }^{1}$ Biomédica, professora doCentro Universitário de Brasília-UniCEUB...E-mail: kikacmvs@yahoo.com.br

Univ. Ci. Saúde, Brasília, v. 3, n. 2, p. 303-312, jul./dez. 2005 
occur preferentially leading to the complete oxidation of the film. The natural PI rubbers, Hevea brasilienses (HB) and Hancornia speciosa (HS), (MG) were characterized by FTIR as being high cis-1,4-polysoprene. The photo-oxidation of NR presented a different kinetic, with induction times smaller than those observed for the cis-PI standards. The faster photodegradation process observed for NR films was assigned to the presence of impurities characterized by the presence of $\mathrm{O}-\mathrm{H}$ and $\mathrm{C}=\mathrm{O}$ str bands in

Key words: photo-oxidations; polysoprenes; FTIR; natural rubbers.

O estudo do efeito da luz UV em borrachas naturais iniciou-se em 1911 por Henry, que confirmou a ocorrência de reações de oxidação. Verificou-se, também, que a absorção de oxigênio depende do comprimento de onda e do tempo de exposição do material à luz ultravioleta. Uma quantidade considerável de trabalhos tem sido desenvolvida nos últimos anos, em vários países, no intuito de compreender os efeitos da degradação da borracha natural (BN), em decorrência da ação do oxigênio do ar e, mais especificamente, compreender o mecanismo responsável pela cisão da cadeia que provoca a diminuição da sua elasticidade. A oxidação de elastômeros e de outros materiais orgânicos na presença de oxigênio elementar é chamada de auto-oxidação e é um processo catalítico.

A pesquisa tem sido realizada no sentido de obter informações sobre as reações químicas que ocorrem e os seus mecanismos. O conhecimento dos processos de desgaste e de envelhecimento pela exposição à luz é de extrema importância. Existe grande interesse da indústria de materiais poliméricos na produção de métodos que previnam a degradação desses materiais. Para tanto, é necessário que se conheçam o aspecto mecânico e as condições que geram o processo de degradação.

A borracha natural é o único polímero natural obtido pela coagulação do látex de algumas espécies vegetais. O látex pode ser definido como uma dispersão coloidal estável de substância polimérica em meio essencialmente aquoso. O principal componente que faz com que seja tão precioso e tão indispensável na vida moderna é o hidrocarboneto de fórmula $\left(\mathrm{C}_{5} \mathrm{H}_{8}\right)_{\mathrm{n}}$, um polímero do isopreno. Além disso, o látex encerra na sua composição outros componentes em proporções menores. Os materiais poliméricos consistem em matéria-prima de vital importância para a produção de computadores, automóveis, aviões, eletrodomésticos em geral, dispositivos médicos e todo tipo de aplicação que necessite de materiais duráveis e resistentes. A figura 1 apresenta, abaixo, o esquema do processo de coagulação do látex e extração por solvente. 


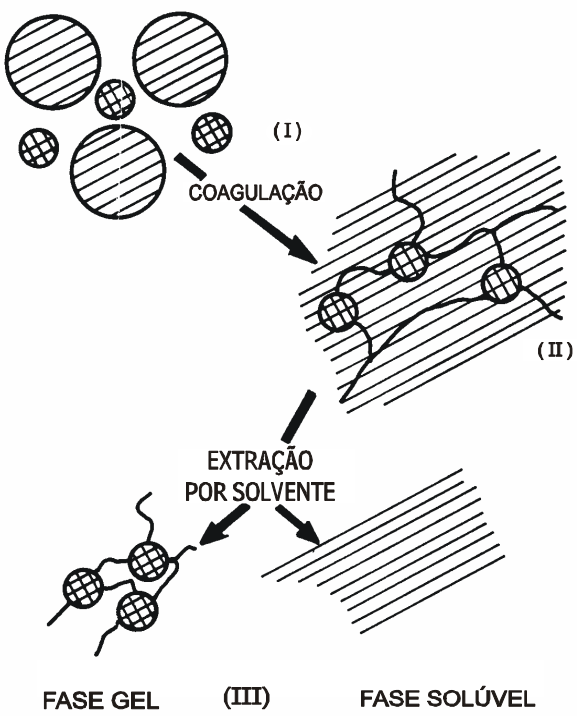

Figura 1 - Representação esquemática do processo de coagulação do látex e extração por solvente.

Antes da coagulação, etapa I da figura 1, o látex apresenta partículas pequenas reticuladas e partículas grandes não reticuladas, envolvidas pelas proteínas contidas no meio dispersivo. O processo de coagulação do látex ocorre pela aglomeração das partículas grandes, formando uma matriz contínua, na qual está contida parte do material reticulado original (III). As partículas pequenas não se aglomeram em decorrência da reticulação. Prolongada extração deste sistema, utilizando solvente apropriado, separa a borracha não- reticulada (fase solúvel) das partículas reticuladas (fase gel), (III).

\section{Elastômeros}

O termo elastômero é utilizado para descrever materiais poliméricos que apresentem grande flexibilidade de cadeia à temperatura ambiente. Os elastômeros podem ser classificados em naturais e sintéticos, apresentando estruturas cis-1,4 polisopreno e trans-1,4 polisopreno.

A BN pode ser extraída de, aproximadamente, 2000 espécies de diferentes famílias. Destas, somente 500 são citadas na literatura como produtoras de borracha em quantidades suficientes para justificar seus estudos. Entre as espécies produtoras de borracha com estrutura cis-1,4-poliisopreno, destaca-se, pela qua- 
lidade e pela quantidade do material: a Hevea brasiliensis (seringueira), pertencente à família Euphorbiaceae, originária da Bacia Amazônica e a Hancornia speciosa (mangabeira), pertencente à tribo Carissae, da família Apocynaceae. A mangabeira é uma árvore presente nos cerrados dos estados centrais do Brasil, chapadas do Nordeste, no Pará e na Amazônia; possui importância econômica, pelo fato de ser espécie frutífera, além de possuir látex com finalidades industriais.

\section{Degradação polimérica}

De acordo com o tipo de iniciação da reação, a degradação de materiais políméricos pode ser classificada como mecânica, química, por radiação de alta energia, biológica, térmica, ultra-sônica, microbiológica e fotoquímica (induzida pela luz, fotodegradação). A degradação mecânica refere-se aos efeitos macroscópicos causados por forças de tração exercidas sobre os polímeros. Processos induzidos exclusivamente sob ação de regentes químicos em contato com o polímero caracterizam a degradação química. Essas reações são dependentes da temperatura, implicando a sobreposição de processos térmicos e químicos. Exemplo disso são os pneus dos automóveis que sofrem atrito com o solo e influência da temperatura. A radiação de alta energia compreende raios- $X$, raios-g, nêutrons, raios-a, etc. A extensão e o caráter da degradação por radiação de alta energia dependem da composição química do material irradiado e do tipo da radiação.

A reação entre enzimas e polímeros caracteriza a degradação biológica. Geralmente, polímeros naturais e sintéticos podem ser atacados por microorganismos de forma química ou mecânica que representam papel eminente na decomposição de materiais orgânicos de todos os tipos, inclusive de polímeros.

Vários actinomicetos isolados da natureza foram capazes de utilizar a BN de $c i s-1,4$ poliisoprenos como fonte de carbono. De acordo com o comportamento de degradação, os representantes desse grupo cresceram somente em contato direto com o substrato da borracha levando à considerável desintegração do material durante o cultivo.

O processo de degradação térmica ocorre por exposição do polímero a altas temperaturas. A deterioração de plásticos em condições ambientais normais é chamada de envelhecimento. Alguns fatores combinados, como a umidade e o calor ou a presença de oxigênio e luz, podem acelerar o processo de deterioração do polímero. A fotodegradação de polímeros ocorre sob a ação de luz visível e ultravioleta, para a qual é necessária a existência de grupos cromóforos na cadeia polimérica, capazes de absorver luz UV-visível.

As reações intramoleculares típicas são: a ciclização e a eliminação. Em polímeros lineares, podem ocorrer reticulações intermoleculares, aumentando a 
massa molar do material. Polímeros lineares do tipo poliolefina decompõem-se formando grandes quantidades de monômeros. Esse processo que ocorre a altas temperaturas é conhecido como despolimerização, que, do ponto de vista cinético, ocorre como na reação em cadeia. A forte dependência da velocidade de despolimerização à temperatura ocorre pelo aumento da velocidade de iniciação e, também, em menos extensão, à cinética de crescimento de cadeia, ambas dependentes da temperatura. $\mathrm{O}$ esquema mostrado na figura 2 apresenta visão geral das reações que ocorrem durante a fotodegradação.

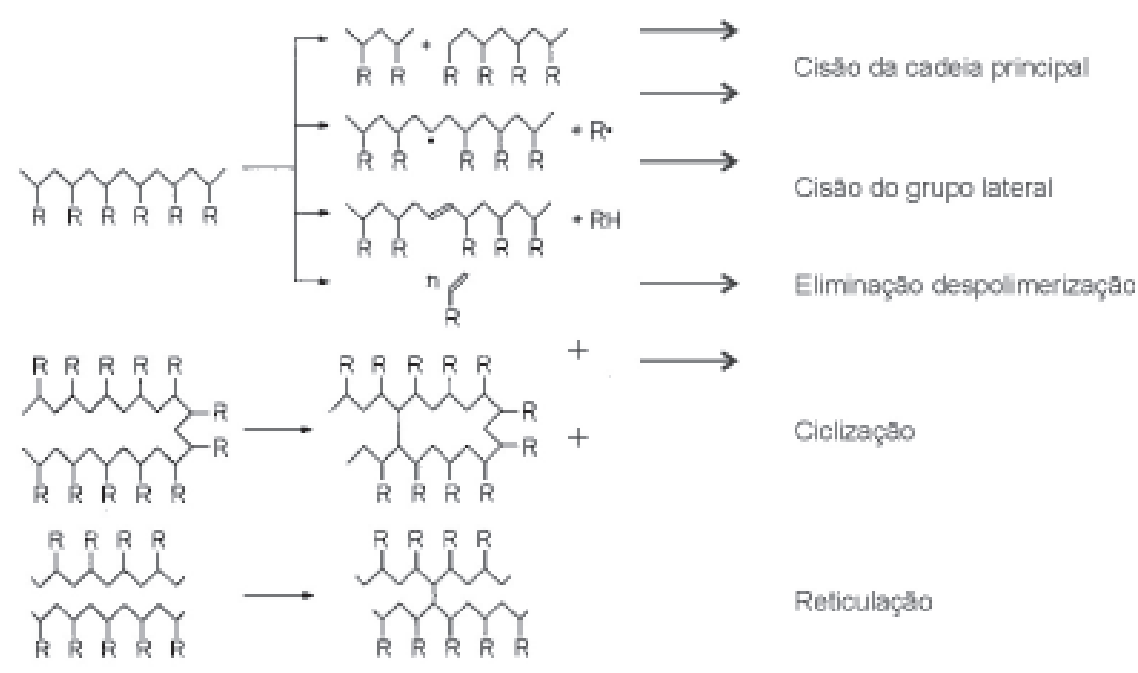

Figura 2 - Reações durante a degradação polimérica.

\section{Fotodegradação de materiais elastoméricos}

Todos os materiais poliméricos, naturais ou sintéticos, são sensíveis a fatores de intempéries, tais como, luz do sol, oxigênio e umidade. A luz solar contém radiações na faixa do ultravioleta que possuem ação destrutiva sobre os materiais poliméricos. Na região do ultravioleta (UV), os comprimentos de onda da radiação eletromagnética, compreendidos entre 100 e $400 \mathrm{~nm}$, estão subdivido em três faixas, UV-A, UV-B e UV-C, conforme apresentado na tabela 1. 
Kárin Santos

Tabela 1 - Comprimentos de ondas eletromagnéticas existentes.

\begin{tabular}{cc}
\hline $\begin{array}{c}\text { Região do espectro } \\
\text { eletromagnético }\end{array}$ & $\begin{array}{c}\text { Comprimento de onda } \\
(\mathrm{nm})\end{array}$ \\
\hline Raios-X & $<100$ \\
UV-C & $100-280$ \\
UV-B & $280-320$ \\
UV-A & $320-400$ \\
Luz visível & $400-700$ \\
\hline
\end{tabular}

O UV-A chega normalmente à superfície terrestre, não sendo absorvido eficientemente por nenhum dos constituintes atmosféricos. Em excesso, pode trazer perturbações à saúde. Normalmente, a proporção de UV-B recebido na superfície do planeta é menor que 1,0\% do total da radiação solar. Mesmo assim, é o principal responsável pela quebra das ligações moleculares e pela conseqüente degradação das películas de tintas e vernizes manifestadas por meio de trincas, perdas de brilho, etc. Na faixa mais energética, o UV-C tem comprimentos de onda mais curtos que $280 \mathrm{~nm}$ e é totalmente absorvido pela camada de ozônio. Os danos causados aos materiais poliméricos pelos raios UV são:

-UV-A: algumas degradações poliméricas;

-UV -B: responsável pela deterioração de muitos polímeros;

- UV-C: pode causar reações irregulares nos polímeros;

As mudanças químicas que ocorrem durante o tratamento de polímeros por fotodegradação devem-se à ruptura de ligações das cadeias principal e lateral, o que pode ser comprovado pela diminuição da massa molar e pela formação de produtos voláteis de baixa massa molar, respectivamente. Na presença de oxigênio, as reações de degradação ocorrem facilmente. A probabilidade da ruptura da cadeia aumenta com o aumento da exposição do material à luz ultravioleta para todos os tipos de ligações, embora as energias de dissociação das ligações sejam diferentes entre si.

\section{Fase experimental}

As BNs foram coletadas e coaguladas naturalmente. Após a coagulação, os elastômeros foram purificados conforme literatura. As BNs foram solubilizadas em tolueno para posterior caracterização. 
Fotodegradação de poliisoprenos

\section{1- Reagentes}

- Látex da borracha natural, LBN, de Hancornia speciosa (mangabeira);

- Látex da borracha natural, LBN, de Hevea brasiliensis (seringueira).

Os látex das BNs de Hancornia speciosa e Hevea brasiliensis foram filtrados sobre gaze, coaguladas espontaneamente, armazenadas sobre proteção da luz a $18^{\circ} \mathrm{C} \mathrm{em} \mathrm{pH} \mathrm{10,0.} \mathrm{Os} \mathrm{demais} \mathrm{reagentes} \mathrm{utilizados} \mathrm{foram} \mathrm{de} \mathrm{grau} \mathrm{analítico.}$

\section{2- Equipamentos}

- Espectometro Bruker Equinox 55S;

- Reator Fotoquímico.

\section{3- Caracterização}

a) Experimento de fotodegradação: $\mathrm{O}$ estudo da fotodegradação da $\mathrm{BN}$ foi realizado em reator fotoquímico, constituído de um tambor rotatório para o acondicionamento das amostras e de duas lâmpadas de $\mathrm{Hg}$, de $8 \mathrm{~W}$ potência cada uma, emitindo em 253, 300 e $350 \mathrm{~nm}$. A distância entre as amostras e a fonte de luz foi de, aproximadamente, $10 \mathrm{~cm}$. A temperatura interna foi controlada e permaneceu em $20^{\circ} \mathrm{C}$ durante a realização de todo o experimento. Os padrões cis poliisopreno foram irradiados por 24 horas, enquanto as amostras de $\mathrm{BN}$ de Hancornia speciosa e Hevea brasiliensis foram irradiadas em intervalo de tempo igual às 6 horas.

b) Espectroscopia de absorção no infravermelho-FTIR (Fourrier Transformed Infrared): Filmes de $\mathrm{BN}$ foram preparados por evaporação sucessiva de alíquotas de solução toluênica das amostras $\mathrm{BN}$ e dos padrões sintéticos sobre placa de $\mathrm{NaCl}$. As amostras foram analisadas por espectroscopia de absorção no infravermelho, FTIR, em espectômetro Bruker Equinox 55S.

c) Resultados das análises de espectroscopia de absorção no infravermelho (FTIR) para filmes de BN de Hancornia speciosa, Hevea brasiliensis e Mimusops globosa: Nos filmes das soluções toluênicas das BNs, preparados em placas de $\mathrm{NaCl}$, observaram-se modificações nos espectros de absorção no infravermelho nas regiões de estiramento $\mathrm{C}=\mathrm{C}\left(1664 \mathrm{~cm}^{-1}\right)$ e $\mathrm{C}=\mathrm{O}\left(1720 \mathrm{~cm}^{-1}\right)$, com o aumento do tempo de exposição à luz ultravioleta, nos comprimentos de onda iguais a 253 e $300 \mathrm{~nm}$. Este comportamento pode ser evidenciado nas figuras 3 e 4 , que apresentam espectros de absorção no infravermelho das regiões de estiramento de $\mathrm{C}=\mathrm{C}\left(1664 \mathrm{~cm}^{-1}\right)$ e $\mathrm{C}=\mathrm{O}\left(1720 \mathrm{~cm}^{-1}\right.$, $)$ nos comprimento de onda igual a 253 e 300nm respectivamente, para as BN de Hancornia speciosa e Hevea brasiliensis. 


\section{Kárin Santos}
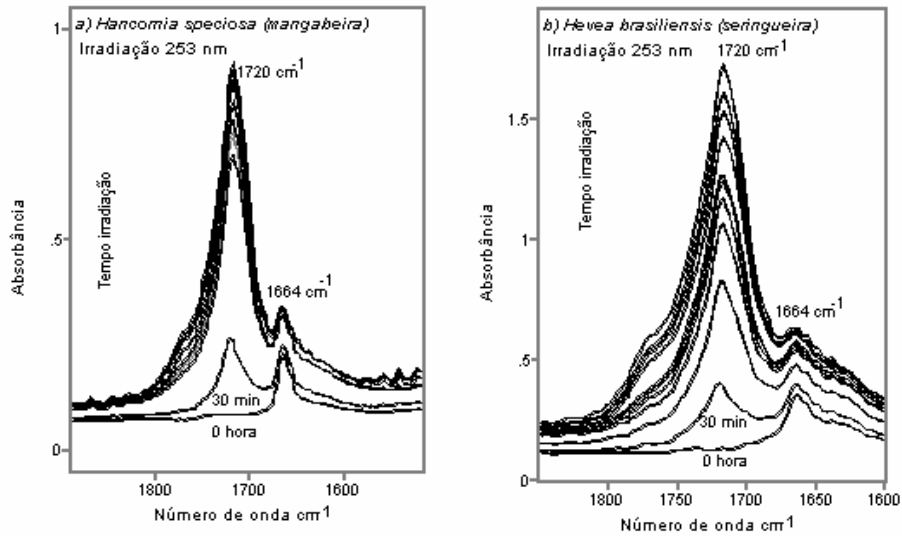

Figura 3 - Espectros de absorção no IV (FTIR) das BNs de Hancornia speciosa (mangabeira) e Hevea brasiliensis (seringueira) no comprimento de onda igual a $253 \mathrm{~nm}$.
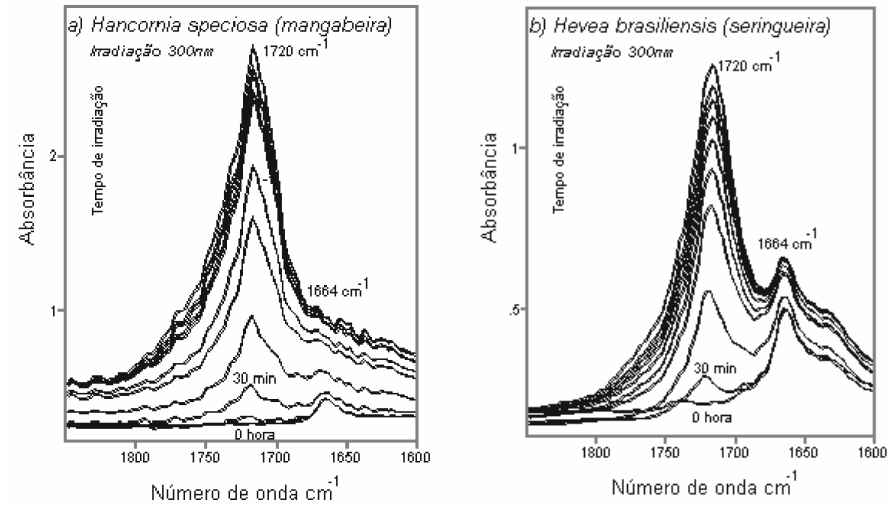

Figura 4 - Espectros de absorção no IV (FTIR) das BNs de Hancornia speciosa (mangabeira) e Hevea brasiliensis (seringueira) no comprimento de onda igual a $253 \mathrm{~nm}$.

Estes resultados mostram comportamento comum a ambas as BN tanto para irradiação em 253 como em $300 \mathrm{~nm}$. O surgimento de banda em ca. $1720 \mathrm{~cm}^{-1}$ aumenta de intensidade com o aumento do tempo de irradiação e a diminuição da intensidade da banda em ca. $1664 \mathrm{~cm}^{-1}$. Outro dado a destacar é que, após 30 minutos de irradiação, em 253 ou $300 \mathrm{~nm}$, os elastômeros já mostravam o surgimento da banda cerca de $1720 \mathrm{~cm}^{-1}$.

O estudo de espectroscopia de absorção no infravermelho (FTIR) para os padrões cis-poliisopreno foi realizado para melhor compreender os mecanismos de ação da radiação UV em BNs e para efeito de comparação com o estudo de 
espectroscopia realizado para as borrachas naturais. Nesta pesquisa, foi utilizado um tempo maior de irradiação, 24 horas, com a finalidade de observar o comportamento da fotodegradação do material após 6 horas de exposição à luz ultravioleta.
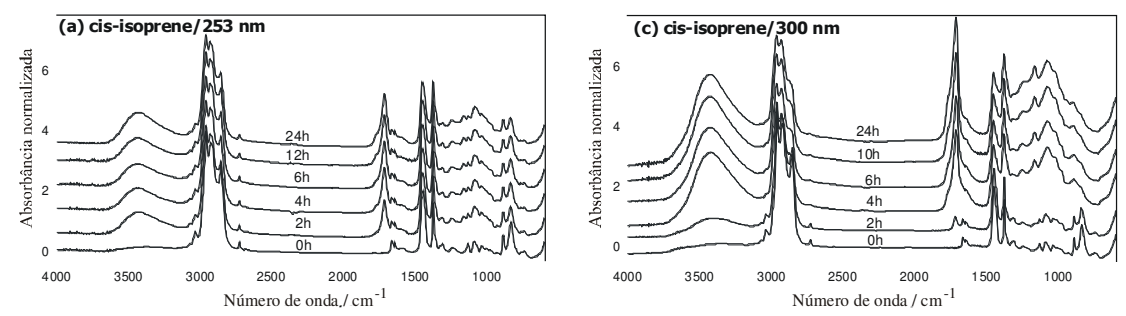

Figura 5 - Espectros FTIR do padrão cis-1,4-poliisoprenos irradiados em intervalos de tempo entre 0 e 24 , horas nos comprimentos de ondas de 253(a) e 300nm(b).

Os modos vibracionais mais afetados pela exposição do padrão de PI à radiação UV são relacionados às insaturações da cadeia polimérica, isto é, o modo de estiramento $\mathrm{n}(\mathrm{C}=\mathrm{C})$ e o modo "wagging" $(=\mathrm{C}-\mathrm{H})$, observados nas regiões espectrais de 1650-1670 e 800-850 $\mathrm{cm}^{-1}$, respectivamente. Os modos $\mathrm{n}(\mathrm{C}=\mathrm{C})$, observados em 1665 e $1668 \mathrm{~cm}^{-1}$, os modos "wagging" (=C-H), observados em 837 e $801 \mathrm{~cm}^{-1}$, para cis e trans-PI, foram selecionados para a avaliação do comportamento de suas intensidades em função do tempo de irradiação. Estes modos vibracionais característicos têm as intensidades diminuídas com a exposição à luz UV, e novas estruturas surgem próximas a 3435,1766 , e $1166 \mathrm{~cm}^{-1}$, as quais correspondem aos modos estiramento $\mathrm{n}(\mathrm{O}-\mathrm{H}), \mathrm{n}(\mathrm{C}=\mathrm{O})$, e $\mathrm{n}(\mathrm{C}-\mathrm{O})$, respectivamente, caracterizando a presença de ácidos carboxílicos. A estrutura de maior intensidade, observada em ca. $1715 \mathrm{~cm}^{-1}$, corresponde ao modo de estiramento $\mathrm{n}(\mathrm{C}=\mathrm{O})$ característico de cetonas. Outros produtos da fotoreação podem ser identificados como o sinal em $880 \mathrm{~cm}^{-1}$, o qual surge após irradiação em 300nm. Esta absorção é atribuída ao modo de estiramento n $(\mathrm{O}-\mathrm{O})$ de peróxidos ou hidroperóxidos. $\mathrm{O}$ mesmo comportamento foi observado para os elastômeros de Hevea brasiliensis e Hancornia speciosa.

\section{Conclusões}

Os resultados apresentados neste trabalho mostraram que o efeito da radiação UV na fotooxidação de poliisoprenos depende do comprimento de onda da fonte de luz utilizada e do tipo de isômero e das condições das borrachas naturais 
e sintéticas. As análises de espectroscopia de absorção no infravermelho (FTIR) mostraram modificações marcantes nas regiões de estiramento correspondente a $\mathrm{n}(\mathrm{C}=\mathrm{C}), \mathrm{n}(\mathrm{C}=\mathrm{O})$ e $\mathrm{n}(\mathrm{OH})$ para os comprimentos de ondas iguais a 253 e $300 \mathrm{~nm}$, com o aumento do tempo de irradiação. Para o comprimento de onda igual a $253 \mathrm{~nm}$, provavelmente, tenha ocorrido reação de reticulação, enquanto, em 300nm, prevaleceu a cisão da cadeia.

\section{Referências}

SANTOS, K.A.M. Suarez, P.A. Z. \& Rubim, J.C., Polymer Degradation and Stability 90(2005) 34-43.

SANTOS, K.A.M. Estudos comparativos dos elastômeros naturais de Hancornia speciosa (mangabeira) e Manihot glaziovii (maniçoba): degradação e fracionamento. Brasília: UnB, 1999. Dissertação de Mestrado.

Estudo da fotodegradação das borrachas naturais (BNS) de Hancornia speciosa, Hevea brasiliensis e Mimusopos globosa e estudo de um método para a determinação de teores de isômeros cis e trans em BNs nativas do cerrado brasileiro.Brasília: UnB, 2004. Tese de Doutorado. 